\title{
The de novo Biosynthesis of Vitamin B6 Is Required for Disease Resistance Against Botrytis cinerea in Tomato
}

\author{
Yafen Zhang, Bo Liu, Xiaohui Li, Zhigang Ouyang, Lei Huang, Yongbo Hong, Huijuan Zhang, Dayong Li, \\ and Fengming Song
}

National Key Laboratory for Rice Biology, Institute of Biotechnology, Zhejiang University, Hangzhou 310058, China

Submitted 28 January 2014. Accepted 18 March 2014.

Vitamin B6 (VB6), an essential cofactor for numerous metabolic enzymes, has recently been shown to act as a potent antioxidant and play important roles in developmental processes and stress responses. However, little is known about the possible function of VB6 in plant disease resistance response against pathogen infection. In the present study, we explored the possible involvement of VB6 in defense response against Botrytis cinerea through functional analysis of tomato VB6 biosynthetic genes. Three de novo VB6 biosynthetic genes (SIPDX1.2, SlPDX1.3, and SIPDX2) and one salvage pathway gene (SISOS4) were identified and the SIPDX1.2, SIPDX1.3, and SIPDX2 genes were shown to encode functional enzymes involved in de novo biosynthesis of VB6, as revealed by complementation of the VB6 prototrophy in yeast snz1 and sno1 mutants. Expression of SIPDX1.2, SIPDX1.3, and SlSOS4 genes was induced by infection with $B$. cinerea. Virus-induced gene silencingmediated knockdown of $S I P D X 1.2$ or $S I P D X 1.3$ but not SIPDX2 and SISOS4 led to increased severity of disease caused by $B$. cinerea, indicating that the VB6 de novo biosynthetic pathway but not the salvage pathway is involved in tomato defense response against $B$. cinerea. Furthermore, the SIPDX1.2- and SIPDX1.3-silenced tomato plants exhibited reduced levels of VB6 contents and reactive oxygen species scavenging capability, increased levels of superoxide anion and $\mathrm{H}_{2} \mathrm{O}_{2}$ generation, and increased activity of superoxide dismutase after infection by $B$. cinerea. Our results suggest that VB6 and its de novo biosynthetic pathway play important roles in regulation of defense response against $B$. cinerea through modulating cellular antioxidant capacity.

Vitamin B6 (VB6) refers to a group of six water-soluble vitamers, including pyridoxal (PL), pyridoxine (PN), pyridoxamine (PM), and their phosphorylated derivatives (pyridoxal 5 -phosphate [PLP], pyridoxamine 5'-phosphate [PMP], and pyridoxine 5 '-phosphate [PNP]), among which PLP is the active form of VB6. The VB6 vitamers are important compounds for all living organisms and function as cofactors in many enzymatic reactions involved in amino acid and fatty acid metabolisms (Drewke and Leistner 2001; Mittenhuber

Corresponding author: F. Song; E-mail: fmsong@zju.edu.cn; Telephone: +1 86-571-88982481; Fax: +1 86-571-88982271.

* The $\boldsymbol{e}$-Xtra logo stands for "electronic extra" and indicates that Figure 5 appears in color online.

(C) 2014 The American Phytopathological Society
2001; Mooney et al. 2009). The de novo biosynthetic pathway requires two protein families, PDX1s and PDX2, which form a PLP synthase complex to synthesize PLP directly. In this pathway, PDX1 catalyzes the final pyridine ring closure whereas PDX2 acts as a glutaminase to supply nitrogen for the PLP heterocycle by hydrolyzing glutamine to glutamate (Dong et al. 2004; Hill and Spenser 1996; Tambasco-Studart et al. 2005, 2007; Wetzel et al. 2004). Alternatively, biosynthesis of the VB6 vitamers can also occur through the salvage pathway, which functions to convert different VB6 vitamers between each other by specific enzymes (McCormick and Chen 1999). Recent studies have demonstrated that both the de novo pathway and the salvage pathway operate in higher plants, as revealed by the identification of a number of genes or enzymes that are involved in VB6 biosynthesis in Arabidopsis at molecular and biochemical levels. There are three $P D X 1$ genes (AtPDX1.1, AtPDX1.2, and AtPDX1.3) and one PDX2 gene $(A t P D X 2)$ which are involved in the de novo biosynthetic pathway (Tambasco-Studart et al. 2005, 2007); while a pyridoxal kinase (AtSOS4), a PNP/PMP oxidase (AtPDX3), and a pyridoxal reductase (AtPLR1) were found to be involved in the salvage pathway (González et al. 2007; Herrero et al. 2011; Lum et al. 2002; Sang et al. 2007, 2011; Shi et al. 2002). Several studies have shown that the de novo pathway is essential in VB6 biosynthesis and is critical in maintaining the balance of VB6 content in plants, because the salvage pathway cannot complement the defect of the de novo pathway deficient plants (Shi and Zhu 2002; Shi et al. 2002; Titiz et al. 2006).

In addition to the importance as enzyme cofactors, VB6 has also been demonstrated to possess antioxidant activity and play important roles in regulating cellular antioxidant defense (Vanderschuren et al. 2013), which may be greater in the antioxidant activity than vitamins C and E (Bilski et al. 2000; Denslow et al. 2005, 2007; Stocker et al. 2003). It was observed that the genes involved in the de novo biosynthetic pathway are upregulated in plant response to various abiotic stresses, including high light, chilling, drought, ozone, and UV-B radiation (Denslow et al. 2005, 2007; Shi et al. 2002; Titiz et al. 2006). Arabidopsis mutants deficient in the $P D X 1$ genes have decreased levels of VB6 and show abnormal growth phenotypes and increased sensitivity to salt and osmotic stress and high light intensity (Chen and Xiong 2005; Havaux et al. 2009; Titiz et al. 2006). In contrast, overexpression of the $P D X$ gene resulted in increased tolerance to oxidative stress (Raschke et al. 2011). During these abiotic stress responses, it was shown recently that VB6 vitamers are efficient quenchers of both singlet oxygen and superoxide anions (Bilski et al. 2000; Denslow et al. 2005). This is evident in the conditional Arabidopsis flu mutant plants, in which VB6 was able to protect flu protoplasts from cell death induced by the release of the singlet oxygen 
under light condition (Danon et al. 2005). On the other hand, the pyridoxal kinase SOS4 and the pyridoxal reductase PLR1 in the salvage pathway were shown to play important roles in response to various abiotic stresses, including salt, cold, and osmotic stress (González et al. 2007; Herrero et al. 2011; Rueschhoff et al. 2013; Shi and Zhu 2002).

Recent evidence also suggests the involvement of VB6 in plant defense response against different types of pathogens. Expression of the $P D X$ genes was induced in tobacco plants after treatment with signaling molecules such as salicylic acid, methyl jasmonate, and ethylene but was decreased after inoculation with an incompatible pathogen, Pseudomonas syringae pv. phaseolicola (Denslow et al. 2005). Excess VB6 in tobacco leaves affected the development of a hypersensitive response (HR) caused by $P$. syringae pv. phaseolicola but increased disease severity caused by a compatible bacterium, $P$. syringae pv. tabaci (Denslow et al. 2005). The Arabidopsis pdxl.3 mutant plants showed an elevated expression level for the $P R-5$ gene (Ristilä et al. 2011). These findings imply that the VB6 vitamers might play important roles in regulating plant defense responses against different types of pathogens. However, direct molecular evidence supporting the hypothesis that VB6 as an antioxidant functions in plant defense is still lacking. The objective of the present study was to explore the possible involvement of VB6 in defense response against Botrytis cinerea by functional analysis of tomato VB6 biosynthetic genes. We found that silencing of SIPDX1.2 or SIPDX1.3 but not SIPDX2 and SlSOS4 led to enhanced susceptibility to $B$. cinerea, reduced levels of VB6 content and total antioxidant capability, increased levels of superoxide anion and $\mathrm{H}_{2} \mathrm{O}_{2}$ generation, and increased activity of superoxide dismutase (SOD) activity after Botrytis infection. Our results suggest that VB6 and its de novo biosynthetic pathway play important roles in regulation of defense response against $B$. cinerea through modulating cellular antioxidant capacity.

\section{RESULTS}

\section{Identification of the VB6 biosynthetic genes in tomato.}

To identify the VB6 biosynthetic genes in tomato, the genomic database (tomato gene mode CDS ITAG release 2.31) was searched by the BlastP program using amino acid sequences of the Arabidopsis PDX1, PDX2, and SOS4 proteins as queries (Shi et al. 2002; Tambasco-Studart et al. 2005, 2007). These searches identified two putative PDX1 genes (Solyc03g120090 and Solyc06g081980), one putative PDX2 gene (Solyc11g069290), and one putative SOS4 gene (Solyc02g091340) in the whole tomato genome. Further searches using the predicted cDNA sequences of these VB6 biosynthetic genes against the Combined Tomato UniGene database were able to retrieve corresponding full-length cDNAs for Solyc03g120090 (SGNU582020), Solyc06g081980 (SGN-U577360 and SGNU590632), and Solyc11g069290 (SGN-U567386) and Solyc02g091340 (SGN-U580571), indicating that these putative VB6 biosynthetic genes are expressed in tomato plants. The sequences of these putative SlPDX1, SlPDX2, and SlSOS4 genes were further confirmed by our reverse-transcriptase polymerase chain reaction (RT-PCR)-based cloning and sequencing. Surprisingly, we only identified two SIPDX1 genes, Solyc03g120090 and Solyc06g081980. This is in agreement with the observation that only two StPDX1 genes were identified but is in contrast to Arabidopsis that has three AtPDX1 members (Mooney et al. 2013; Tambasco-Studart et al. 2005). Alignment of protein sequences with AtPDX1s revealed that Solyc03g120090 has 67.5\% identity to AtPDX1.2 (61.3 and $65.2 \%$ identity to AtPDX1.1 and AtPDX1.3, respectively) and Solyc06g081980 has $90.6 \%$ identity to AtPDX1.3 (87.4 and
60.2\% identity to AtPDX1.1 and AtPDX1.2, respectively); thus, we named Solyc03g120090 and Solyc06g081980 for SIPDX1.2 and SIPDX1.3, respectively. The SIPDX1.3 protein contains a characteristic sequence of LPVVNFAAGGVATPA DAAL (UPF0019), while the SIPDX1.2 protein lacks this characteristic sequence, which is consistent with those for AtPDX1.2 and AtPDX1.3 (Tambasco-Studart et al. 2005). The putative PDX2 gene Solyc11g069290, named SIPDX2, encodes a protein showing $70.9 \%$ identity to AtPDX2, and containing almost the entire signature sequence of this protein family ([GA]LI[LIV]PGGEST[STA], UPF0030) (Tambasco-Studart et al. 2005). The putative SOS4 gene Solyc02g091340, named SlSOS4, encodes a protein showing $82.5 \%$ identity to AtSOS4, and containing conserved motifs that may be involved in substrate binding or catalysis (Shi et al. 2002).

\section{Biochemical characterization of SIPDX1s and SIPDX2.}

To determine whether the cloned SIPDX1.2, SIPDX1.3, and SIPDX2 encode functional enzymes, the coding sequence of these genes were cloned into pYES2, an $S$. cerevisiae expression vector under the control of a GAL1 promoter for highlevel inducible expression in yeast by galactose, and were tested for their ability to complement the SNZ1 (functional PDX1 homolog) and SNO1 (functional PDX2 homolog) disruption mutants, BQS1037 and MML21, respectively (RodríguezNavarro et al. 2002; Tambasco-Studart et al. 2005). When grown on synthetic complete (SC) medium supplemented with VB6, the BQS1037 strains transformed with the SIPDX1.2 or $S I P D X 1.3$ genes and the MML21 strain transformed with gene SIPDX2 showed growth rates similar to those of strains transformed with the $S$. cerevisiae $S N Z 1$ or $S N O 1$ genes and with the corresponding empty vectors, respectively (Fig. 1). In the absence of VB6, growth defects were observed in the SIPDX1.2- or SIPDX1.3-transformed BQS1037 strains, which grew faster than the empty-vector-transformed strain but slower than the S. cerevisiae SNZ1-transformed strain (Fig. 1A). Meanwhile, the SIPDX1.3-transformed BQS1037 strain showed better growth than the SIPDX1.2-transformed BQS1037 strain (Fig. 1A). These data suggest that the cloned SIPDX1.2 and SIPDX1.3 restored the VB6 prototrophy in yeast. Likewise, when grown on SC medium without VB6, the SIPDX2-transformed MML21 strain showed similar growth to the strain transformed with the $S$. cerevisiae SNO1 gene but enhanced growth to the strain transformed with the empty vector (Fig. 1B), indicating that the cloned SIPDX2 has a homological biochemical function as the SNO1 gene in the de novo VB6 biosynthetic pathway.

\section{Silencing of SlPDX1.2 and SlPDX1.3 led to enhanced susceptibility to $B$. cinerea.}

To explore the involvement of VB6 in tomato defense response, we examined whether blocking of VB6 biosynthesis by virus-induced gene silencing (VIGS)-mediated silencing of the genes involved in the de novo and salvage pathways affected the defense response of tomato plants to fungal infection. For this purpose, we made Tobacco rattle virus (TRV)mediated VIGS constructs for SIPDX1.2, SIPDX1.3, SIPDX2, and SlSOS4 genes. Standard VIGS protocol was performed and the VIGS-infiltrated plants were used for various assays 3 weeks later, when the bleaching phenotype in the pTRV-phytoene desaturase (PDS)-infiltrated plants appeared. Silencing efficiency was examined by quantitative (q)RT-PCR analysis of the transcript abundance of the target genes in the pTRVSIPDX1.2-, pTRV-SIPDX1.3-, pTRV-SIPDX2-, or pTRVSISOS4-infiltrated plants and compared with those in the pTRV- $\beta$-glucuronidase (GUS)-infiltrated negative control plants. Results revealed that the transcript levels of the SIPDX1.2, $S I P D X 1.3$, SlPDX2, and SlSOS4 genes were significantly re- 
duced in the VIGS-infiltrated plants compared with those in the pTRV-GUS-infiltrated control plants and the silencing efficiencies were $>75 \%$ (Fig. 2A). In our study, we observed that the SIPDX1.3-silencied plant showed ab abnormal leaf developmental phenotype (e.g., yellowish and small leaves), indicating a possible function for SIPDX1.3 in development. This is similar to the previous observations that the Arabidopsis $p d x 1.3$ mutant plants had reduced leaf size and developed yellowish leaves due to a reduction of chlorophyll content (Titiz et al. 2006; Wagner et al. 2006). No significant morphological phenotypes were observed in the SIPDX1.2-, SIPDX2-, or SISOS4-silenced plants compared with the control plants.

A detached leaf assay was first used to examine the disease phenotype of the VIGS-infiltrated plants after inoculation with $B$. cinerea. Detached leaves from 5-week-old VIGS-infiltrated plants were inoculated by dropping $2.5 \mu \mathrm{l}$ of spore suspension $\left(1 \times 10^{5}\right.$ spores $\left./ \mathrm{ml}\right)$ onto the leaf surface and disease development was evaluated at 3 days postinoculation (dpi). Under our disease assay conditions, a typical disease symptom (e.g., necrotic lesions) was observed in the leaves from all VIGS-infiltrated plants but the lesions in the leaves from the SIPDX1.2or SIPDX1.3-silenced plants expanded much more rapidly compared with others (Fig. 3A). At 3 dpi, the lesion sizes in leaves from the pTRV-SIPDX1.2- or pTRV-SIPDX1.3-infiltrated plants were approximately 7.5 and $7.1 \mathrm{~mm}$, respectively; 30 to $37 \%$ larger than those on the controls (approximately 5.5 $\mathrm{mm}$ ) (Fig. 2B). However, the lesions on the leaves from the pTRV-SIPDX2- or pTRV-SISOS4-infiltrated plants, measuring approximately 5.9 and $5.8 \mathrm{~mm}$, respectively, were similar in size to the controls (Fig. 2B). A whole-plant inoculation method was further used to confirm the disease phenotypes observed above in the VIGS-infiltrated plants after inoculation by foliar spraying with spore suspension of $B$. cinerea. The pTRVSIPDX1.2- or pTRV-SIPDX1.3-infiltrated plants showed more significant diseases at 3 dpi compared with the control plants (Fig. 3B) and most of the plants (>90\%) died and were rotten at $7 \mathrm{dpi}$; however, no significant difference in disease severity and progress was observed among the pTRV-SIPDX2-, pTRVSISOS4-, and pTRV-GUS-infiltrated plants during our experimental period. Analysis of the transcript for the $B$. cinerea BcActinA gene as indicative of the rate of fungal growth in planta showed similar results compared with analysis of the disease phenotypes. At $3 \mathrm{dpi}$, growth of $B$. cinerea in leaf tissues of the pTRV-SIPDX1.2- and pTRV-SIPDX1.3-infiltrated plants was approximately 28.6- and 25.4-fold higher compared with the controls (Fig. 2C). No significant difference in fungal growth was observed in the pTRV-SIPDX2-, pTRV-SISOS4-, and pTRV-GUS-infiltrated plants (Fig. 2C). These results indicate that silencing of SIPDX1.2 and SIPDX1.3 resulted in increased susceptibility against $B$. cinerea and that $S I P D X 2$ and SlSOS4 may have no contribution to disease resistance against $B$. cinerea in tomato.

\section{Expression of VB6 biosynthetic genes and changes of VB6 content in response to $B$. cinerea.}

The enhanced susceptibility against $B$. cinerea in the SIPDX1.2- and SIPDX1.3-silenced plants led us to hypothesize that $B$. cinerea may interfere with the VB6 biosynthesis in tomato plants during its infection process. Therefore, we first examined the expression patterns of the SIPDX1.2, SIPDX1.3, and SISOS4 genes in wild-type tomato plants after infection with B. cinerea. Expression levels of SIPDX1.2, SIPDX1.3, and SISOS4 were comparable at different time points in mockinoculated plants but were significantly induced with different patterns in B. cinerea-inoculated plants (Fig. 4A). After inoculation with $B$. cinerea, expression levels of SIPDX1.2 increased significantly at 24 and $48 \mathrm{~h}$ postinoculation (hpi): more than three times higher than those in the mock-inoculated plants (Fig. 4A). Expression levels of the SIPDX1.3 and SISOS4 also increased approximately 0.6 to 1.8 times higher than those in the mock-inoculated plants (Fig. 4A). These results indicate that expression of the genes involved in the de novo pathway

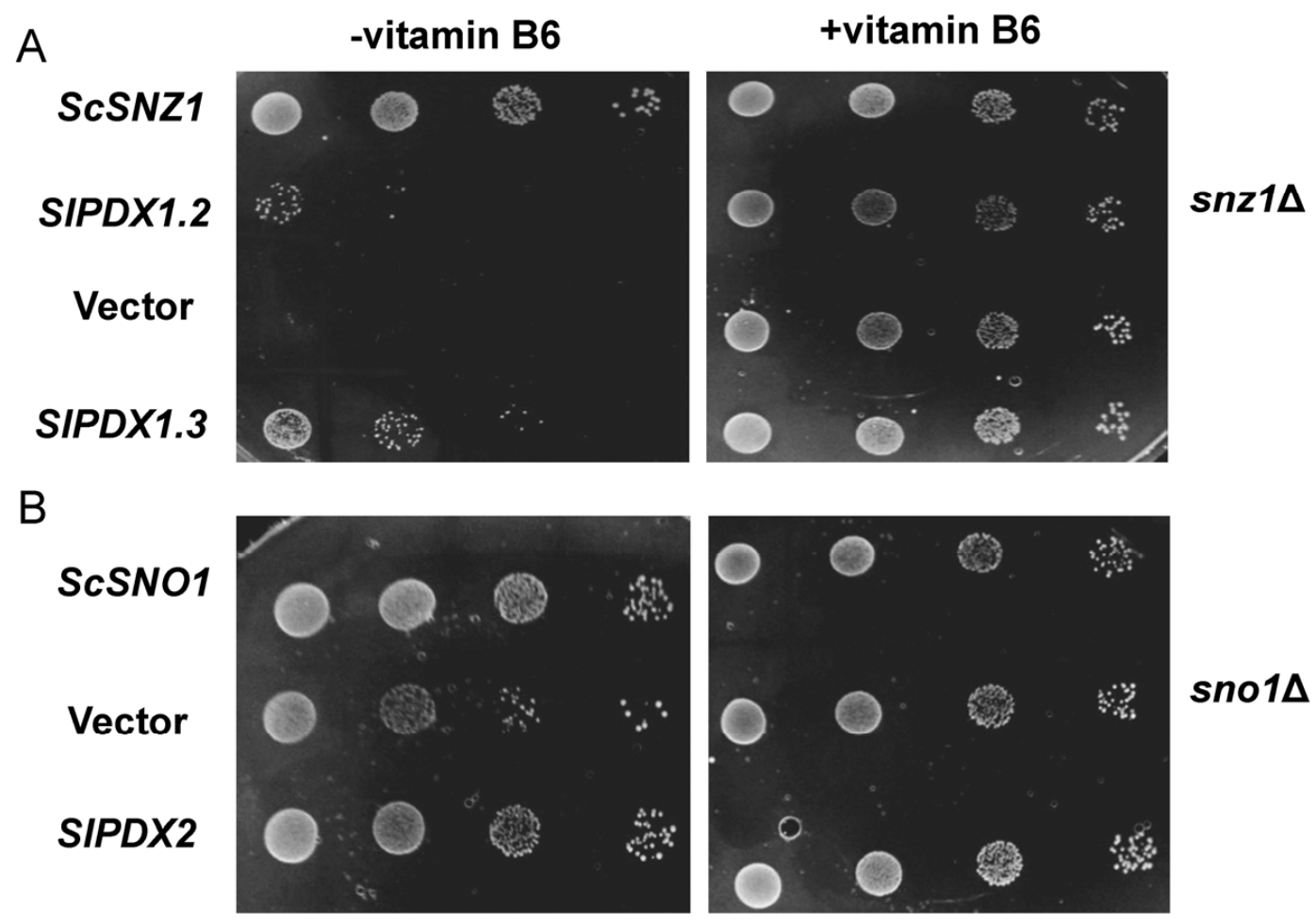

Fig. 1. Functional complementation of tomato SlPDX1s and SlPDX2 genes in yeast with defects in SNZ1 (functional PDX1 homolog) and SNO1 (functional PDX2 homolog). Left: Growth of A, 6-day-old BQS1037 and B, 4-day-old MML21 in the absence of VB6. Right: Growth of A, 3-day-old BQS1037 and B, MML21 in the presence of VB6. Each row represents a serial 10-fold dilution from a starter culture with an optical density at $600 \mathrm{~nm}$ of 0.5 . 
as well as in the salvage pathway can be induced by infection of $B$. cinerea.

To examine whether the $B$. cinerea-induced expression of the VB6 biosynthetic genes affects the biosynthesis of VB6 in tomato plants, we analyzed the changes in VB6 contents in the pTRV-SIPDX1.2- and pTRV-SIPX1.3-infiltrated plants before and after infection with $B$. cinerea. Before inoculation with $B$. cinerea, the pTRV-SIPDX1.2-infiltrated plants contained VB6 content in leaf tissues similar to that in the control plants; however, the VB6 content in leaf tissues of the pTRV-SIPDX1.3infiltrated plants was significantly reduced, showing only approximately $50 \%$ of the VB6 content in the control plants (Fig. 4B). At 48 hpi with $B$. cinerea, VB6 content was increased markedly in the control plants, resulting in an increase of $53 \%$ compared with the content before inoculation (Fig. 4B). Although the VB6 contents in the pTRV-SIPDX1.2- and pTRVSIPX1.3-infiltrated plants were also increased after infection with B. cinerea (Fig. 4B), the increase was $<23 \%$ compared with the contents before inoculation and was less evident than that observed in the control plants. These data indicate that silencing of the SIPDX1.3 gene decreased the capability of the de novo VB6 biosynthesis but silencing of either the SIPDX1.2 or SIPDX1.3 gene can reduce the B. cinerea-triggered VB6 biosynthesis in tomato plants.

\section{Increased accumulation of reactive oxygen species in the $S I P D X 1.2$ - and SIPDX1.3-silenced plants after Botrytis sp. infection.}

Considering that reactive oxygen species (ROS) has been implicated in susceptible responses of plants against necrotrophic fungal pathogens such as $B$. cinerea (Veronese et al. 2006) and that VB6 plays important roles in regulating cellular antioxidant defense (Vanderschuren et al. 2013), we asked whether decreased VB6 content in the SIPDX1.2- or SIPDX1.3-silenced plants affected the accumulation of ROS in response to $\mathrm{B}$. $\mathrm{Ci}$ nerea. We determined ROS content by in situ detection of superoxide anion and quantitative measurement of $\mathrm{H}_{2} \mathrm{O}_{2}$ and compared the accumulation of ROS in the pTRV-SIPDX1.2-, pTRV-SIPDX1.3-, and pTRV-GUS-infiltrated plants before and after infection with B. cinerea (Fig. 5). Without infection, no significant accumulation of superoxide anion and $\mathrm{H}_{2} \mathrm{O}_{2}$ in the leaves was observed among the pTRV-SIPDX1.2-, pTRVSIPDX1.3-, and pTRV-GUS-infiltrated plants, indicating that silencing of SIPDX1.2 or SIPDX1.3 did not affect the accumulation of superoxide anion and $\mathrm{H}_{2} \mathrm{O}_{2}$ in tomato plants. In con-

Fig. 2. Silencing of SIPDX1.2 and SIPDX1.3 resulted in increased severity of disease caused by Botrytis cinerea. Two-week-old tomato seedlings were infiltrated with agrobacteria carrying pTRV-SIPDX1.2, pTRVSIPDX1.3, pRTV-SIPDX2, pTRV-SISOS4, or pTRV-GUS (as a negative nonsilenced control [NS]) constructs, and inoculation was carried out at 3 weeks after virus-induced gene silencing infiltration by $\mathbf{B}$, dropping spore suspension on detached leaves or $\mathbf{C}$, foliar spraying with spore suspension. A, Silencing efficiency for target genes in silenced plants. The silencing efficiency was calculated by comparing the transcript levels of SIPDX1.2, SIPDX1.3, SIPDX2, and SISOS4 in pTRV-SIPDX1.2-, pTRV-SIPDX1.3-, pRTV-SIPDX2-, or pTRV-SISOS4-infiltrated plants to those in the NS plants, which were set as 1 . B, Lesion sizes on selected leaves in detached leaf inoculation assays at 3 days postinoculation (dpi). Lesion sizes were measured on a minimum of 30 leaves in each experiment. C, Growth of $B$. cinerea in inoculated plants from whole-plant inoculation experiments. Fungal growth in planta was assumed at 3 dpi by analyzing the transcript level of $B$. cinerea BcActinA gene by quantitative reverse-transcriptase polymerase chain reaction using the SlActin gene as an internal control. Relative fungal growth was shown as folds of transcript levels of BcActin compared with SlActin. Data presented are the means \pm standard deviation from three independent experiments, and different letters above the columns indicate significant differences at $P<0.05$. trast, infection with $B$. cinerea accelerated markedly the accumulation of superoxide anion and $\mathrm{H}_{2} \mathrm{O}_{2}$ in the leaves but the accumulation levels in the pTRV-SIPDX1.2- and pTRVSIPDX1.3-infiltrated plants were more evident and higher than that in pTRV-GUS-infiltrated plants (Fig. 5). At $24 \mathrm{hpi}$, accumulation of $\mathrm{H}_{2} \mathrm{O}_{2}$ in pTRV-SIPDX1.2- and pTRV-SIPDX1.3infiltrated plants showed an increase of 25 to $30 \%$ over that in the pTRV-GUS-infiltrated plants (Fig. 5B). No difference in the ROS accumulation was observed between the pTRVSIPDX1.2- and pTRV-SIPDX1.3-infiltrated plants with or without inoculation. These data indicate that silencing of SIPDX1.2 and SIPDX1.3 can promote the accumulation of superoxide and $\mathrm{H}_{2} \mathrm{O}_{2}$ in tomato plants when infected with $B$.
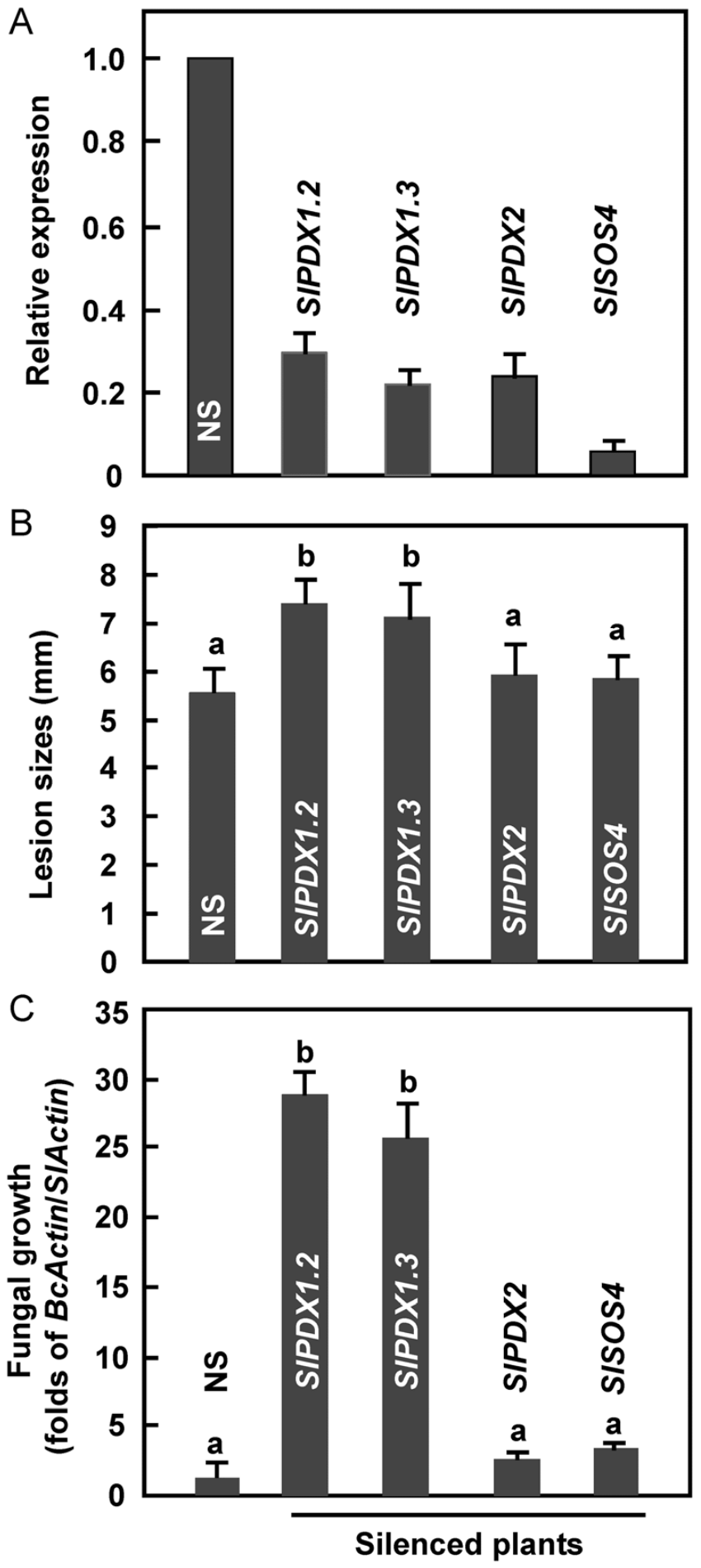

Vol. 27, No. 7, 2014 / 691 
cinerea, implying a relationship between decreased VB6 content and increased accumulation of ROS in the SIPDX1.2- and SIPDX1.3-silenced plants.

\section{Changes of ROS scavenging capability}

\section{in the SIPDX1.2- and SIPDX1.3-silenced plants.}

Increased ROS accumulation in the SIPDXI.2- and SIPDX1.3-silenced plants after infection with $B$. cinerea led us to examine whether silencing of SIPDX1.2 or SIPDX1.3 affects the capability of ROS scavenging system in tomato plants. We first analyzed the changes of ROS scavenging capability (RSC), representing the ability to scavenger superoxide anion, in the pTRV-SIPDX1.2- and pTRV-SIPDX1.3-infiltrated plants and compared them with those in the pTRV-GUS-infiltrated plants. Without inoculation with $B$. cinerea, silencing of either the SIPDX1.2 or SIPDX1.3 gene decreased the RSC in tomato plants (the mock-inoculation control and at 0 hpi the inoculation treatment) compared with that in the pTRV-GUS-
A

B
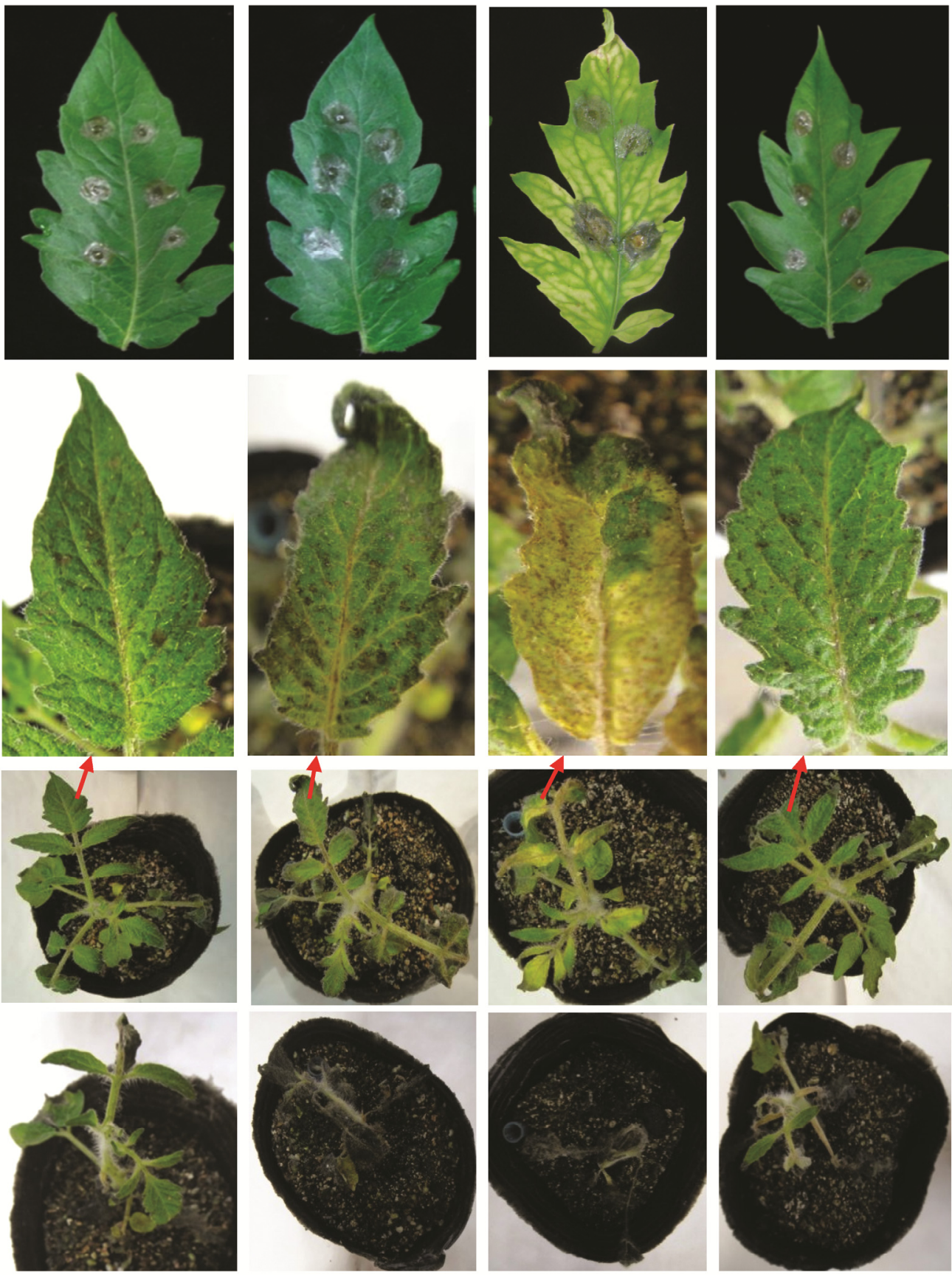

NS

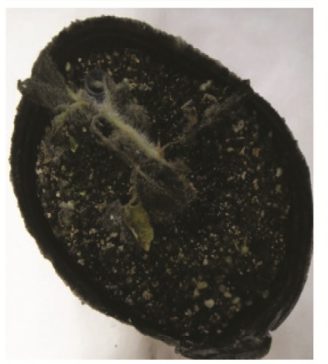

SIPDX1.2

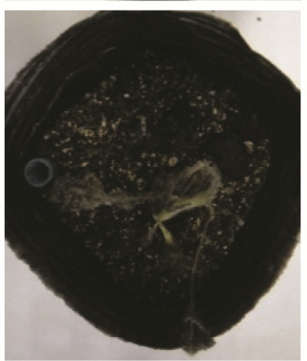

SIPDX1.3

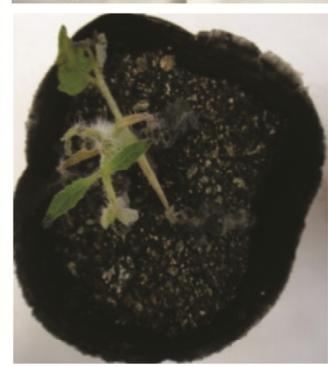

SIPDX2
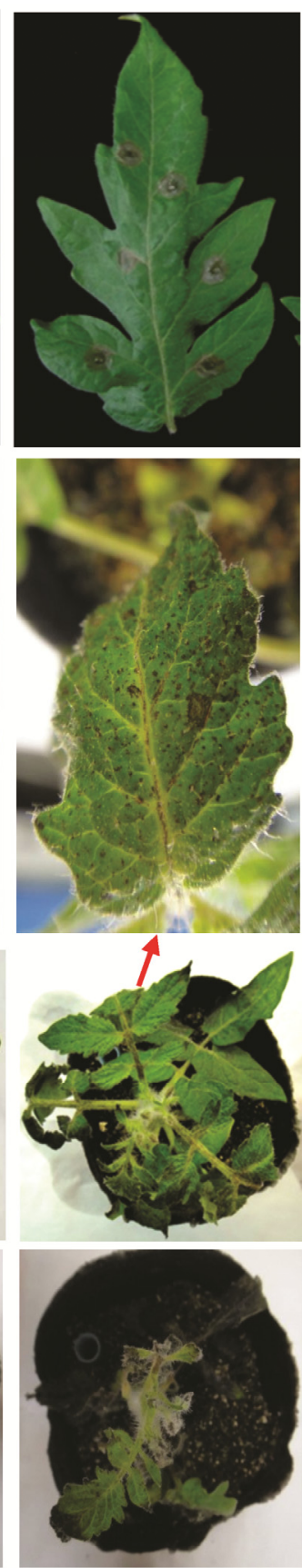

SISOS4

\section{Silenced plants}

Fig. 3. Botrytis cinerea-caused disease phenotype in SIPDX1.2-, SIPDX1.3-, SIPDX2-, and SISOS4-silenced tomato plants. Two-week-old tomato seedlings were infiltrated with agrobacteria carrying pTRV-SIPDX1.2, pTRV-SIPDX1.3, pRTV-SIPDX2, pTRV-SISOS4, or pTRV-GUS (as a negative nonsilenced control [NS]) constructs, and inoculation was carried out at 3 weeks after virus-induced gene silencing infiltration by A, dropping spore suspension on detached leaves or B, foliar spraying with spore suspension. A, Disease symptoms on detached leaves at 3 days postinoculation (dpi). B, Disease phenotype on representative leaves and whole plants at 3 and 7 dpi, respectively. Red arrows indicate the representative diseased leaves in the inoculated whole plants. 
infiltrated plants (Fig. 6). The RSC increased significantly at 24 hpi with $B$. cinerea in all plants, indicating that infection of $B$. cinerea can trigger the response of RSC in plants (Fig. 6). However, the increase patterns of the RSC differed among the pTRV-SIPDX1.2-, pTRV-SIPDX1.3-, and pTRV-GUS-infiltrated plants when compared with their corresponding mockinoculated control plants. The RSC in the B. cinerea-infected pTRV-GUS-infiltrated plants increased almost onefold $(98.86 \%)$ over that in the mock-inoculated plants; however, the RSC in the $B$. cinerea-infected pTRV-SIPDX1.2- and pTRV-SIPDX1.3infiltrated plants showed approximately 65.71 to $68.32 \%$ increase compared with their corresponding mock-inoculated plants (Fig. 6). These data reflect a reduction of the RSC increase in the SIPDX1.2- and SIPDX1.3-silenced plants compared with the increase in the pTRV-GUS-infiltrated plants after infection, suggesting that silencing of either the SIPDX1.2 or
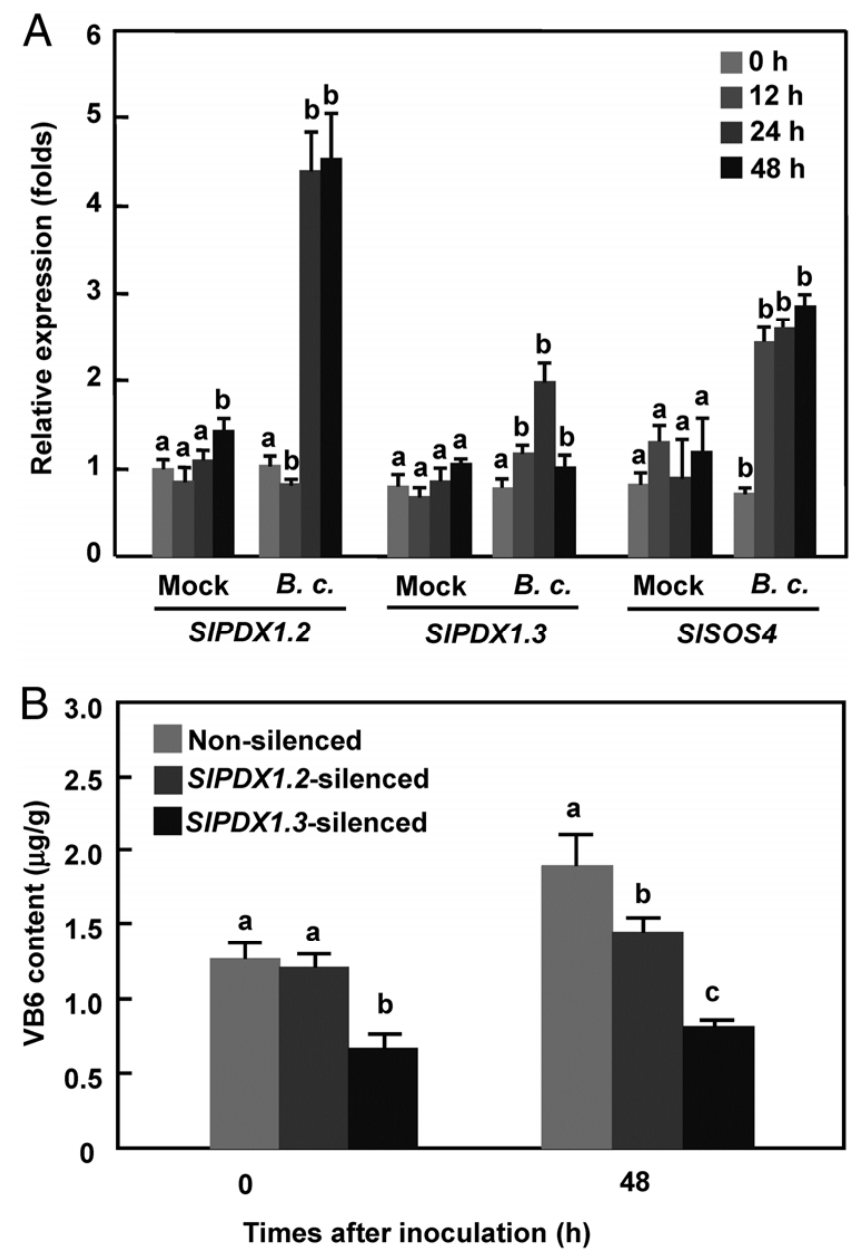

Fig. 4. Expression of SIPDX1.2 and SIPDX1.3 and changes in VB6 contents after Botrytis infection. A, Expression patterns of SIPDX1.2 and SIPDX1.3 in tomato plants after infection with Botrytis cinerea. Fourweek-old tomato plants were inoculated by foliar spraying with spore suspension of $B$. cinerea and leaf samples were collected at indicated time points after inoculation. Gene expression was analyzed by quantitative reverse-transcriptase polymerase chain reaction with actin gene SlActin as an internal control. B, Changes of VB6 contents in SIPDX1.2- and SIPDX1.3-silenced plants after Botrytis infection. Two-week-old tomato seedlings were infiltrated with agrobacteria carrying pTRV-SIPDX1.2, pTRV-SIPDX1.3, or pTRV-GUS (as a negative nonsilenced control) constructs and inoculated with spore suspension of $B$. cinerea at 3 weeks after virus-induced gene silencing infiltration. Leaf samples were collected at 0 and $48 \mathrm{~h}$ after inoculation and were subject to analyze total VB6 content by yeast bioassay. Data presented in B are the means \pm standard deviation from three independent experiments and different letters above the columns indicate significant differences at $P<0.05$.
SlPDX1.3 gene in tomato suppresses the B. cinerea-triggered RSC.

We next analyzed the changes in activity of SOD and catalase (CAT) and contents of reduced or oxidized glutathione (GSH or GSSG, respectively), key components of the ROS scavenging system, in the pTRV-SIPDX1.2- and pTRVSIPDX1.3-infiltrated plants and compared them with those in the pTRV-GUS-infiltrated plants. Without inoculation with $B$. cinerea, similar activity of SOD and CAT and contents of GSH and GSSG were observed, indicating that silencing of SIPDX1.2 or SIPDX1.3 itself had no effect on these components of the ROS scavenging system in tomato plants (Figs. 7 and 8). The activity of SOD and CAT was increased but the GSH content was reduced after inoculation with $B$. cinerea (Figs. 7 and 8), similar to previous observations in tomato plants after infection with $B$. cinerea (Kuźniak and Skłodowska
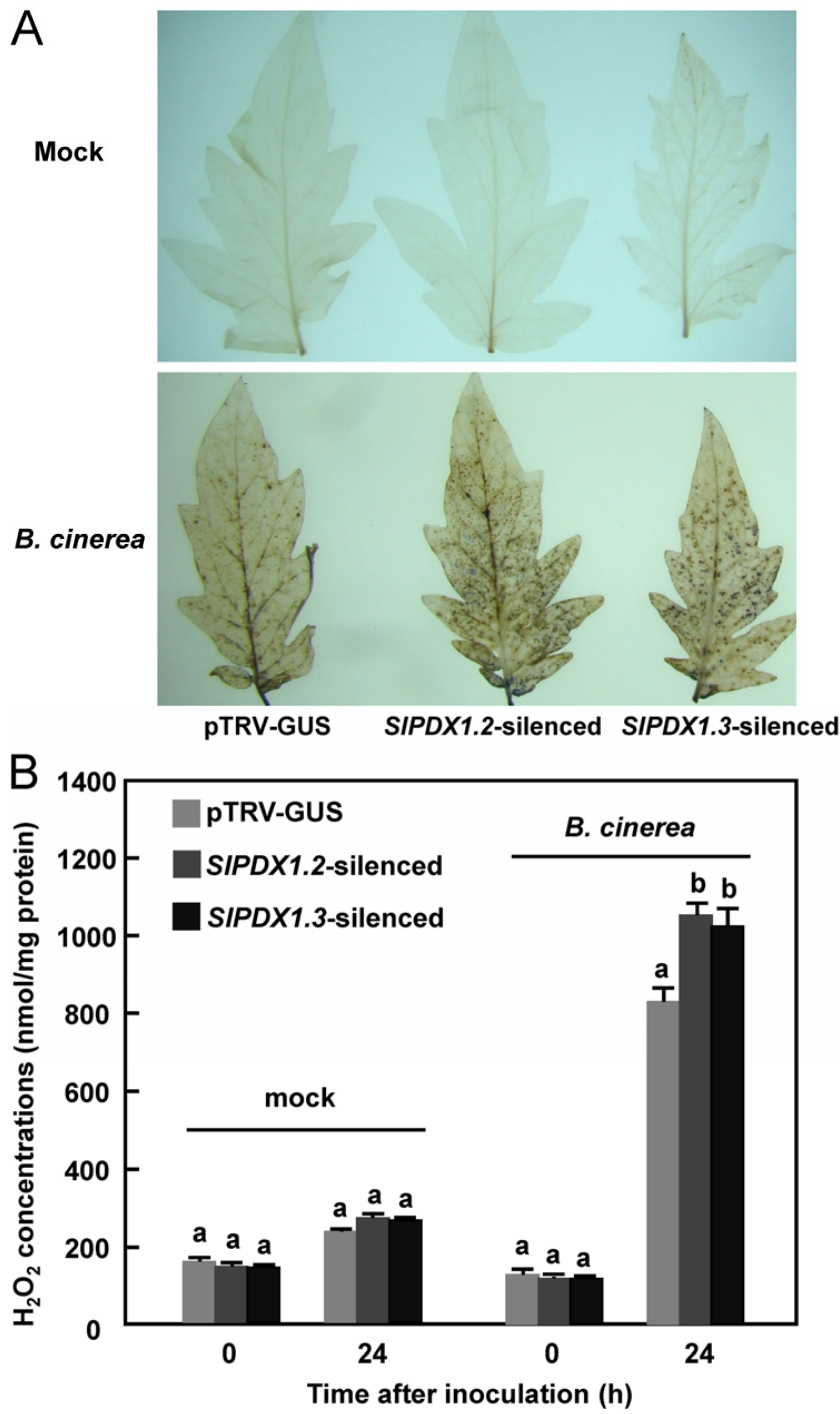

Fig. 5. Increased accumulation of reactive oxygen species (ROS) in the SIPDX1.2- and SIPDX1.3-silenced plants after Botrytis infection. Twoweek-old tomato seedlings were infiltrated with agrobacteria carrying pTRV-SIPDX1.2, pTRV-SIPDX1.3, or pTRV-GUS (as a negative nonsilenced control) constructs and foliar spraying inoculated with spore suspension of Botrytis cinerea at 3 weeks after virus-induced gene silencing infiltration. Leaf samples were collected 0 and $24 \mathrm{~h}$ after inoculation and were subjected to $\mathbf{A}$, in situ detection of superoxide anion or $\mathbf{B}$, quantitative measurement of $\mathrm{H}_{2} \mathrm{O}_{2}$ accumulation. Data presented in $\mathrm{B}$ are the means \pm standard deviation from three independent experiments and different letters above the columns indicate significant differences at $P<$ 0.05 . 
2004, 2005a and b). At 24 hpi, no significant difference in the CAT activity and the GSH and GSSG contents was observed between the pTRV-SIPDX1.2- or pTRV-SIPDX1.3-infiltrated plants and the pTRV-GUS-infiltrated plants (Figs. 7B and 8A). However, the SOD activity was significantly higher in the pTRV-SIPDX1.2- or pTRV-SIPDX1.3-infiltrated plants, showing an increase of $30 \%$ compared with that in the pTRV-GUSinfiltrated plants (Fig. 7A). Notably, no difference in RSC, activity of SOD and CAT and contents of GSH or GSSG was observed between the pTRV-SIPDX1.2- and pTRV-SIPDX1.3infiltrated plants with or without inoculation.

\section{DISCUSSION}

Functions of VB6 as antioxidant compounds in plant abiotic stress responses has been well documented recently (Bilski et al. 2000; Danon et al. 2005; Denslow et al. 2005; González et al. 2007; Havaux et al. 2009; Herrero et al. 2011; Raschke et al. 2011; Rueschhoff et al. 2013; Shi and Zhu 2002; Titiz et al. 2006). Although a previous study has implicated the involvement of VB6 in HR and disease development (Denslow et al. 2005), direct molecular evidence of the function of VB6 in plant defense response against pathogen attack remains elusive. In this study, we presented molecular evidence that the de novo VB6 biosynthetic pathway but not the salvage pathway is involved in tomato defense response against $B$. cinerea because silencing of tomato SIPDX1.2 and SIPDX1.3 but not SlSOS4 led to increased disease severity. Combination of the data from our molecular and biochemical analyses led to a conclusion that VB6 and its de novo biosynthetic pathway have a positive function in regulation of defense response through modulating cellular antioxidant capacity. These findings extend our knowledge of the biological function of VB6 and its biosynthesis, which integrate into the plant defense response against pathogen attack.

The de novo pathway contributes predominantly to the biosynthesis of VB6 and, hence, affects the total VB6 content, whereas the salvage pathway involves the interconversion

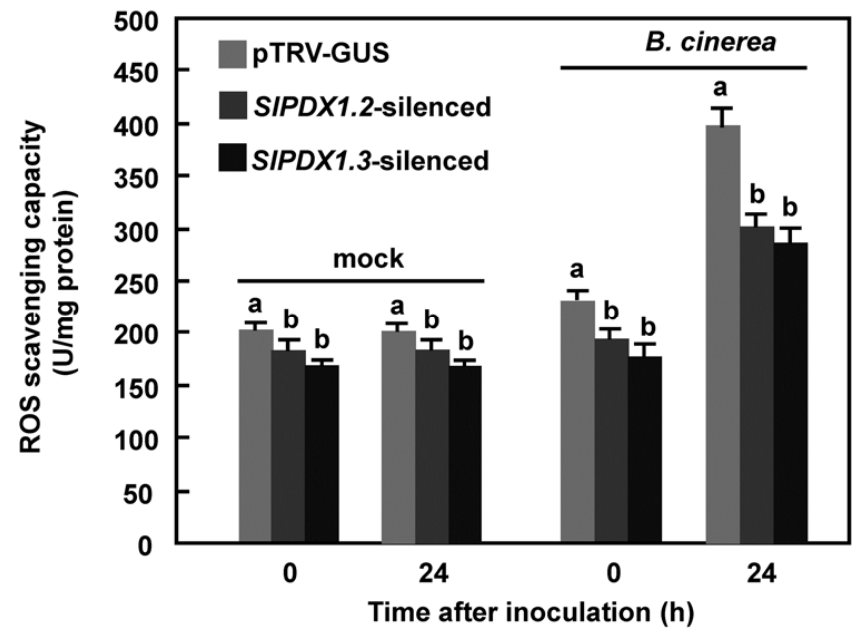

Fig. 6. Reduced capacity of reactive oxygen species (ROS) scavenger in SIPDX1.2- and SIPDX1.3-silenced plants. Two-week-old tomato seedlings were infiltrated with agrobacteria carrying pTRV-SIPDX1.2, pTRVSIPDX1.3, or pTRV-GUS (as a negative nonsilenced control) constructs and foliar spraying inoculated with spore suspension of Botrytis cinerea at 3 weeks after virus-induced gene silencing infiltration. ROS scavenger capacity represents the overall ability of the samples to scavenger superoxide anion generated from the xanthine and xanthine oxidase system in the reactions. Data presented are the means \pm standard deviation from three independent experiments and different letters above the columns indicate significant differences at $P<0.05$. among the different VB6 vitamers (Dong et al. 2004; Hill and Spenser 1996; McCormick and Chen 1999; Wetzel et al. 2004). In the present study, blocking of the de novo VB6 biosynthetic pathway by silencing of $S I P D X 1.2$ or $S I P D X 1.3$ resulted in an increased level of disease caused by $B$. cinerea but blocking of the salvage pathway by silencing of the SlSOS4 gene did not alter the disease phenotype (Figs. 2 and 3). Similar results were also found in Arabidopsis: mutations in AtPDX1.2 or AtPDX1.3 led to increased levels of diseases caused by $\mathrm{B}$. $\mathrm{ci}$ nerea and $P$. syringae pv. tomato DC3000 (Y. Zhang and F. Song, unpublished data). Furthermore, silencing of SIPDX1.3 but not SIPDX1.2 resulted in a decreased level of VB6 in leaf tissues of tomato plants (Fig. 4). This is in agreement with previous findings that the Arabidopsis AtPDX1.3, together with AtPDX1.1, interacts with AtPDX2 to form a complex that directly synthesizes the cofactor form of VB6 (TambascoStudart et al. 2005; Wagner et al. 2006). Surprisingly, silencing of the SIPDX2 gene, a key gene in the de novo pathway, did not alter the phenotype of disease caused by $B$. cinerea in our repeated experiments (Figs. 2 and 3). Taken together, it is likely that the de novo pathway of VB6 biosynthesis is essential for plant disease resistance against different types of patho-
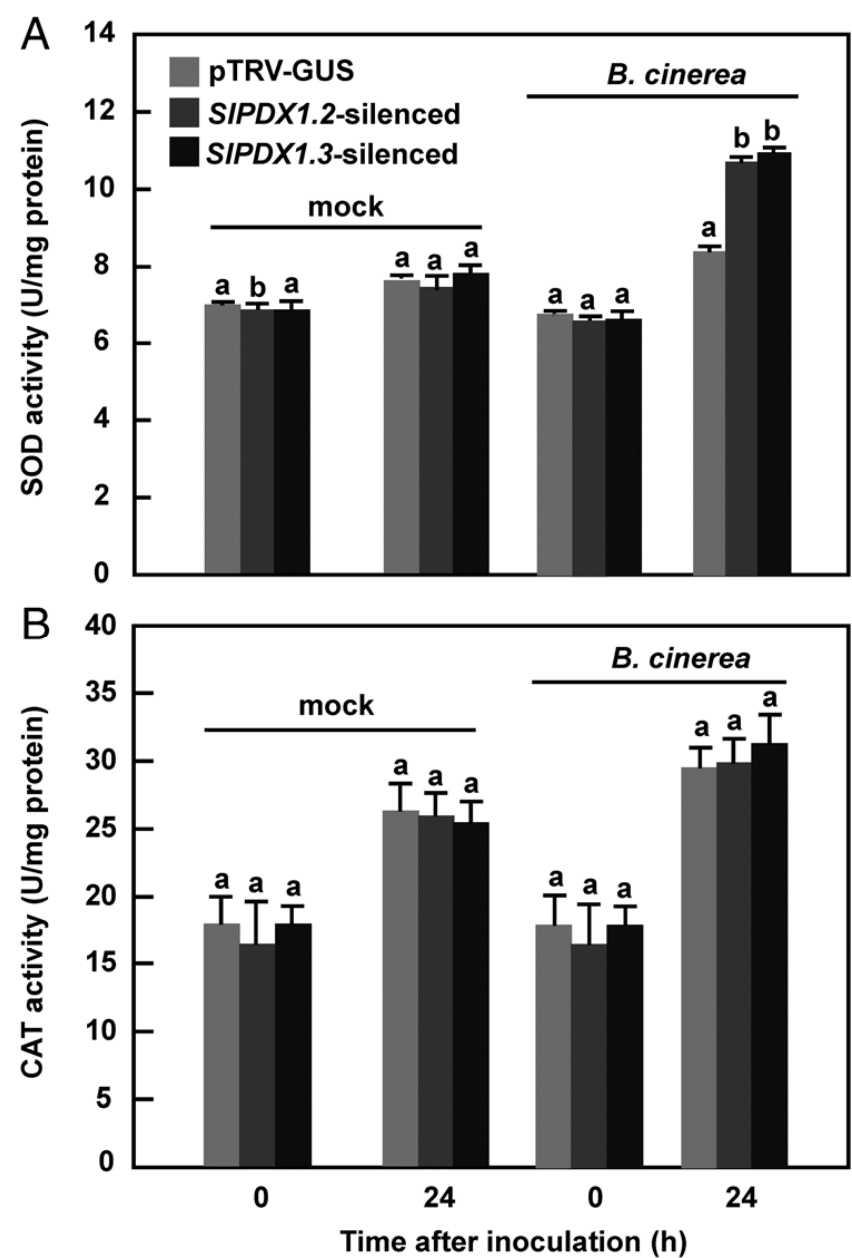

Fig. 7. Changes in superoxide dismutase (SOD) and catalase (CAT) activity in SIPDX1.2- and SIPDX1.3-silenced plants. Two-week-old tomato seedlings were infiltrated with agrobacteria carrying pTRV-SIPDX1.2, pTRVSIPDX1.3, or pTRV-GUS (as a negative nonsilenced control) constructs and foliar spraying inoculated with spore suspension of Botrytis cinerea at 3 weeks after virus-induced gene silencing infiltration. Activities of A, SOD and B, CAT were analyzed using commercial kits. Data presented are the means \pm standard deviation from three independent experiments and different letters above the columns indicate significant differences at $P<0.05$. 
gens. Previous studies with Arabidopsis mutants have shown that the de novo pathway is essential and critical in biosynthesis and maintaining the balance of VB6 content in plants, and that the salvage pathway cannot compensate for the defect of the de novo pathway-deficient plants (Tambasco-Studart et al. 2005; Titiz et al. 2006). On the other hand, we also found that the Arabidopsis sos 4 mutant plants showed increased levels of diseases caused by $P$. syringae pv. tomato DC3000 and $B$. cinerea in another study (Y. Zhang and F. Song unpublished data), indicating the involvement of the salvage pathway in disease resistance. Therefore, it is also likely that the requirement of the salvage pathway for disease resistance differs among different plant species (e.g., increased level of disease caused by $B$. cinerea in the Arabidopsis sos 4 plants but no alteration in disease phenotype in the tomato SISOS4-silenced plants).

The de novo pathway of VB6 involves only two proteins, PDX1 and PDX2, which function together as a glutamine aminotransferase to catalyze the direct formation of the cofactor vitamer (Tambasco-Studart et al. 2005). Surprisingly, only two SIPDX1 genes, SIPDX1.2 and SIPDX1.3, were identified in the tomato genome, which is similar to the findings in potato (Mooney et al. 2013). Thus, it is likely that the Solanaceae plants encode for two but not three $P D X 1$ genes. Expression of SIPDX1.2, SIPDX1.3, and SISOS4 genes in tomato plants was induced significantly in response to infection by $B$. cinerea (Fig. 4A). We also found that infection by $B$. cinerea increased the VB6 content in tomato plants (Fig. 4B). It was previously found that the transcript levels of tobacco PDX1 and PDX2 genes decreased following inoculation with an incompatible pathogen, $P$. syringae pv. phaseolicola, which induced an HR (Denslow et al. 2005). These data indicate that the de novo biosynthetic pathway responds differentially to infection by different types of pathogens. This is supported by the fact that silencing of either the SIPDX1.2 or SIPDX1.3 gene can reduce the $B$. cinerea-triggered VB6 biosynthesis in tomato plants (Fig. 4B) and facilitate the development of disease caused by B. cinerea (Figs. 2 and 3). However, our results are in contrast to a previous observation that excessive VB6 $(100 \mathrm{mM})$ injected into tobacco leaves led to increased disease severity caused by a compatible bacterium (Denslow et al. 2005).

Like the Arabidopsis AtPDX1.2 that is not involved directly in VB6 biosynthesis (Tambasco-Studart et al. 2005; Wagner et al. 2006), the tomato SIPDX1.2 seems also not associated with the de novo pathway of VB6 biosynthesis because silencing of SIPDX1.2 did not alter the VB6 content in tomato leaf tissues (Fig. 4B). Surprisingly, silencing of SIPDX1.2 did result in increased level of disease caused by B. cinerea (Figs. 2 and 3), indicating a role for SIPDX1.2 in disease resistance against this pathogen. Recently, it was found that the Arabidopsis AtPDX1.2 assembles in planta with either of the other two PDX1 proteins, probably forming higher-order AtPDX1.2containing complexes, and, thus, affects the de novo VB6 biosynthesis (Leuendorf et al. 2010). In this regard, mechanisms for how SIPDX1.2 regulates the de novo VB6 biosynthesis and, thus, affects defense response in tomato remain to be explored further.

It is well documented that ROS play important roles in the establishment of infection by some necrotrophic pathogens such as B. cinerea (Mengiste 2012). Recently, it was shown that accumulation of anthocyanins in tomato fruit can extend shelf life and reduce susceptibility to $B$. cinerea through increasing total antioxidant capacity (Zhang et al. 2013), indicating the importance of antioxidant capacity in resistance to $B$. cinerea. When compared with those in the control plants, increased disease level and increased accumulation of $\mathrm{H}_{2} \mathrm{O}_{2}$ and superoxide anion after infection with $B$. cinerea was ob- served in the SIPDX1.2- and SIPDX1.3-silenced plants (Figs. 2,3 , and 5). This is similar to the previous observations in some Arabidopsis mutants, which showed increased disease severity and higher levels of ROS (Mengiste et al. 2003; Veronese et al. 2006). Markedly, accompanying the $B$. cinereatriggered increased levels of ROS represented by accumulation of $\mathrm{H}_{2} \mathrm{O}_{2}$ and superoxide anion, is the pronounced decline of RSC in the SIPDX1.2- and SIPDX1.3-silenced plants compared with the control plants after infection with $B$. cinerea (Figs. 6 to 8). This pronounced decline of RSC might be caused by multiple factors in the complicated ROS scavenging system in plants. The augmented increase of SOD activity over that in the control plants accelerated enzymatic conversion of the superoxide anion to $\mathrm{H}_{2} \mathrm{O}_{2}$ and led to further accumulation of $\mathrm{H}_{2} \mathrm{O}_{2}$ in the SIPDX1.2- and SlPDX1.3-silenced plants, which showed CAT activity similar to that in the control plants (Fig. 7). B. cinerea infection-induced decrease of GSH contents and, thus, GSH/GSSG ratios was previously reported in tomato plants (Kuźniak and Skłodowska 2004, 2005a). However, no significant difference in $B$. cinerea-induced decrease of the GSH contents was observed between the SIPDX1.2- and SIPDX1.3-silenced plants and the control plants after B. cinerea
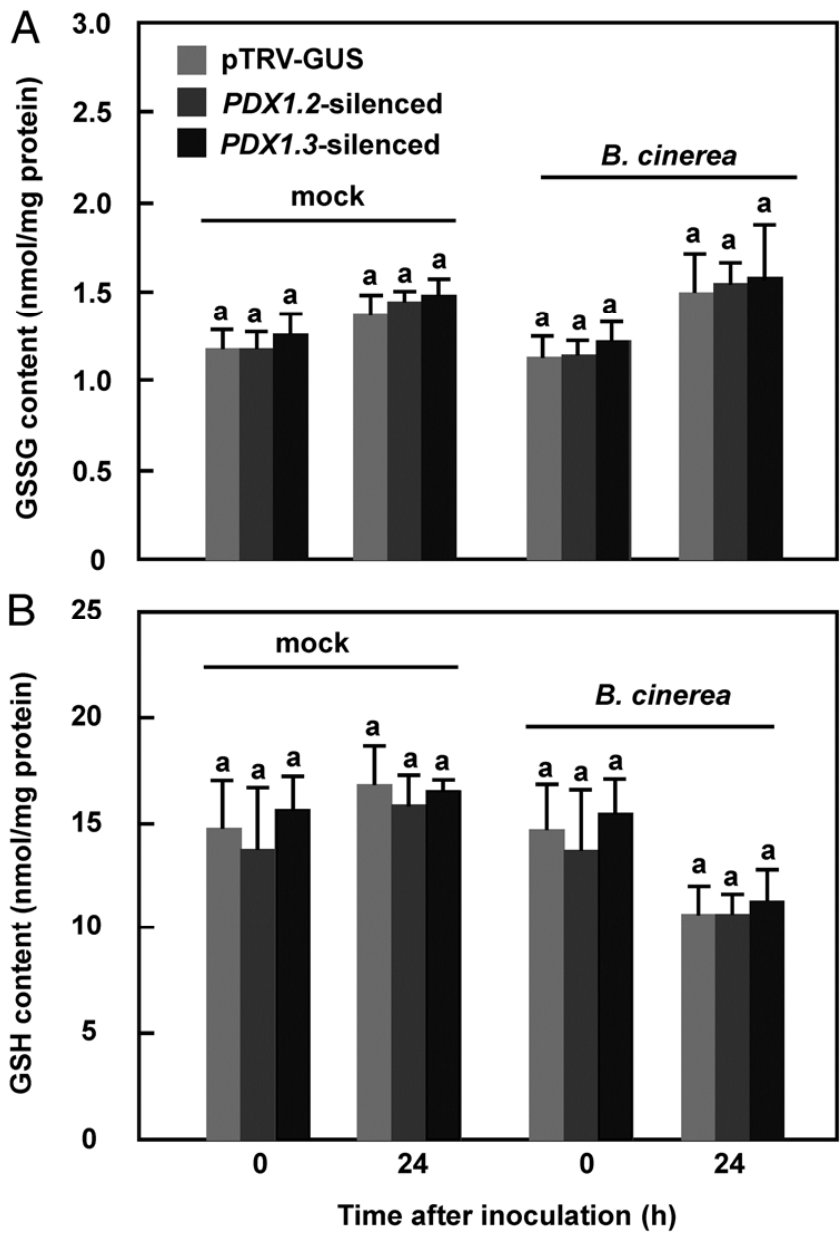

Fig. 8. Changes in reduced and oxidized glutathione (GSH and GSSG, respectively) contents in SIPDX1.2- and SIPDX1.3-silenced plants. Twoweek-old tomato seedlings were infiltrated with agrobacteria carrying pTRV-SIPDX1.2, pTRV-SIPDX1.3, or pTRV-GUS (as a negative nonsilenced control) constructs and foliar spraying inoculated with spore suspension of Botrytis cinerea at 3 weeks after virus-induced gene silencing infiltration. GSSG and GSH were analyzed using commercial kits. Data presented are the means \pm standard deviation from three independent experiments and different letters above the columns indicate significant differences at $P<0.05$. 
infection (Fig. 8), indicating that the change of the GSH and GSSG contents is unlikely a cause that led to the pronounced decline of RSC in the SIPDX1.2- and SIPDX1.3-silenced plants. On the other hand, VB6 has been demonstrated to be an efficient antioxidant capable of quenching ROS (Bilski et al. 2000; Denslow et al. 2005, 2007; Stocker et al. 2003). Coincidence between the pronounced declines in VB6 contents and RSC levels and the augmented increase in ROS accumulation in the SIPDX1.2- and SIPDX1.3-silenced plants, when compared with those in the control plants, after infection with $B$. cinerea establishes a connection between VB6 as an antioxidant and the cellular RSC. Further direct biochemical and physiological evidence supporting this hypothesis needs to be investigated.

\section{MATERIALS AND METHODS}

\section{Plant growth and sample collection.}

Tomato (Solanum lycopersicum) 'Suhong 2003' and 'microTom' were used for different purposes in the experiments. Tomato seed were scarified on moist filter paper in petri dishes for 2 days and the sprouted seed were transferred into a mix- ture of perlite/vermiculite/plant ash (1:6:2) and grown in a growth room at $22^{\circ} \mathrm{C}$ under a regime of $16 \mathrm{~h}$ of light $(350 \mu \mathrm{mol}$ $\mathrm{s}^{-1} \cdot \mathrm{m}^{-2}$ photons $\mathrm{m}^{-2} \mathrm{~s}^{-1}$ ) and $8 \mathrm{~h}$ of darkness. For gene expression and biochemical analyses, 4-week-old tomato plants were inoculated by foliar spraying with a spore suspension of $\mathrm{B}$. $\mathrm{Ci}$ nerea (discussed below) or with a similar volume of $4 \%$ maltose and $1 \%$ peptone solution as mock-inoculation controls. Inoculated plants were covered with a transparent plastic film and kept in a growth chamber under conditions similar to those mentioned above. Leaf samples were collected at indicated time points after inoculation.

\section{Cloning of the tomato VB6 biosynthetic genes and gene expression analysis.}

The tomato genomic database was searched to identify the VB6 biosynthetic genes using Arabidopsis PDX and SOS4 genes as queries. The predicted SIPDX and SISOS4 genes and their available cDNA sequences were used to design gene-specific primers (Table 1) to amplify full-length cDNAs by RTPCR. Expression and silencing efficiency of the VB6 biosynthetic genes were analyzed by qRT-PCR using gene-specific primers (Table 1). The PCR reactions were normalized against

Table 1. List of primers used in this study

\begin{tabular}{|c|c|c|}
\hline Primers & Sequences $\left(5^{\prime}-3^{\prime}\right)$ & Size (bp) \\
\hline \multicolumn{3}{|l|}{ Cloning } \\
\hline SIPDX1.2- $1 \mathrm{~F}$ & ATG GAA GAA GAC GGT GCC GTT ACA & 918 \\
\hline SIPDX1.2-1R & CAT CAG TAG TTC TCA TCA CTA ACA & $\ldots$ \\
\hline SIPDX1.3-1F & ATG GCC GGA AGT GGT GTC GTT ACA & 930 \\
\hline SIPDX1.3-1R & TCA CTC AGA ACG ATT GGC ATA CCT & $\ldots$ \\
\hline SIPDX2-1F & ATG GTT GTG GGT GTT CTT GC & 756 \\
\hline SIPDX2-1R & CTA CTG GTA TAT GGG AAA ATC & \\
\hline SISOS4-1F & ATG TTT GTT TCT CCA CTC ATC A & 1,020 \\
\hline SISOS4-1R & TTA GTT ATA TCT TTT AGC TCT A & $\ldots$ \\
\hline \multicolumn{3}{|c|}{ Virus-induced gene silencing (VIGS) } \\
\hline SIPDX1.2-VIGS-1F & 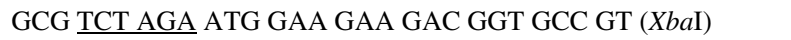 & 438 \\
\hline SIPDX1.2-VIGS-1R & ATA CTC GAG TAA CGC TTC TCC AAG ATC TC $(X h o \mathrm{I})$ & \\
\hline SIPDX1.3-VIGS-1F & GCG $\overline{\text { TCT AGA }}$ ATG GCC GGA AGT GGT GTC GT ( $\mathrm{Xba \textrm {I }})$ & 398 \\
\hline SIPDX1.3-VIGS-1R & 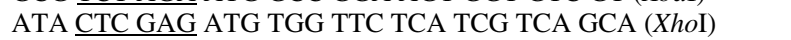 & \\
\hline SIPDX2-VIGS-1F & GCG $\overline{\text { TCT AGA }}$ TGA GAA AGC CAG AGC AGT TGC (XbaI) & 554 \\
\hline SIPDX2-VIGS-1R & ATA $\overline{\text { CTC GAG }}$ ACT GTG CCA TCG AGT ATC TGC (XhoI) & $\ldots$ \\
\hline SISOS4-VIGS-1F & GCG TCT AGA CCA GGT GCT TGA AGC AAT TGC A $(X b a \mathrm{I})$ & 493 \\
\hline SISOS4-VIGS-1R & 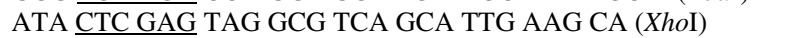 & $\ldots$ \\
\hline SIPDS-VIGS-1F & CGG TCT AGA GGC ACT CAA CTT TAT AAA CC (XbaI) & 409 \\
\hline SIPDS-VIGS-1R & CGG $\overline{\text { GGA TCC }}$ CTT CAG TTT TCT GTC AAA CC (BamHI) & $\ldots$ \\
\hline GUS-VIGS-1F & CGG TCT AGA ACC TGG GTG GAC GAT ATC AC (XbaI) & 396 \\
\hline GUS-VIGS-1R & CGG $\overline{\text { GGA TCC }}$ GTG CAC CAT CAG CAC GTT AT (BamHI) & $\ldots$ \\
\hline \multicolumn{3}{|c|}{ Quantitative reverse-transcriptase polymerase chain reaction } \\
\hline SIPDX1.2-rt-1F & GAT GCA GCT GGG TTG TGA TG & 144 \\
\hline SIPDX1.2-rt-1R & TCC AAA CCA CTG CTA GCC GC & $\ldots$ \\
\hline SIPDX1.3-rt-1F & CAT GTG CGT TCC GTT ATG GG & 140 \\
\hline SIPDX1.3-rt-1R & TGA ACC ACA GGG AGC CTA CC & 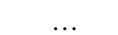 \\
\hline SISOS4-rt-1F & GCG TAT TTC ACG GGA ACT GG & 104 \\
\hline SISOS4-rt-1R & CTT GAG ACA GCA AGC TCT GC & $\ldots$ \\
\hline SIPDX2-rt-1F & CAA AGC TTC GGA ACG CGT TCA A & 113 \\
\hline SIPDX2-rt-1R & GTC AAT GAG TAG CCA TTT GAC C & $\ldots$ \\
\hline SlActin-1F & AGG CAC ACA GGT GTT ATG GT & 177 \\
\hline SlActin-1R & AGC AAC TCG AAG CTC ATT GT & $\ldots$ \\
\hline BcActin-1F & TCC AAG CGT GGT ATT CTT ACC C & 117 \\
\hline BcActin-1R & TGG TGC TAC ACG AAG TTC GTT G & $\ldots$ \\
\hline \multicolumn{3}{|l|}{ Yeast complementation } \\
\hline ScSNZ1-1F & ATA GGA TCC ATG ACT GGA GAA GAC TTT AAG A (BamHI) & 894 \\
\hline ScSNZ1-1R & GCG TCT AGA TCA CCA CCC AAT TTC GGA AAG T (XbaI) & $\ldots$ \\
\hline ScSNO1-1F & ATA GGA TCC ATG CAC AAA ACC CAC AGT ACA A (BamHI) & 675 \\
\hline ScSNO1-1R & GCG $\overline{\text { TCT AGA }}$ TTA ATT AGA AAC AAA CTG TCT G $(X b a \mathrm{I})$ & $\ldots$ \\
\hline SIPDX1.2-y-1F & GCG GAG CTC ATG GAA GAA GAC GGT GCC GT (SacI) & 918 \\
\hline SIPDX1.2-y-1R & 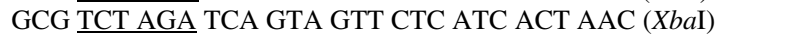 & $\ldots$ \\
\hline SIPDX1.3-y-1F & GCG $\underline{\text { GAG CTC }}$ ATG GCC GGA AGT GGT GTC GT (SacI) & 930 \\
\hline SIPDX1.3-y-1R & 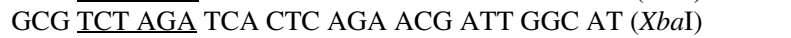 & \\
\hline SIPDX2-y-1F & ATA $\overline{\text { GGA TCC }}$ ATG GTT GTG GGT GTT CTT GC (BamHI) & 756 \\
\hline SIPDX2-y-1R & GCG $\underline{\text { TCT AGA }}$ CTA CTG GTA TAT GGG AAA ATC (XbaI) & $\ldots$ \\
\hline
\end{tabular}


a tomato actin gene (Table 1) for comparative analysis. Total RNA was extracted using TRIZOL and the first-strand cDNA was obtained with RNA PCR kit (Ver. 3.0; TaKaRa, Dalian, China) according to the manufacturer's protocols. qRT-PCR analyses were repeated with three biological replicates by using SYBR Green PCR master mix kit (TaKaRa) in a CFX96 real-time PCR detection system (Bio-Rad, Hercules, CA, U.S.A.), according to the manufacturer's instructions. Relative expression was calculated using $2^{-\Delta \Delta \mathrm{CT}}$ method, as described (Livak and Schmittgen 2001).

\section{VIGS assays.}

For construction of VIGS vectors, gene-specific primers (Table 1) with restriction enzyme sites were designed to amplify fragments of 300 to 500 bp for SIPDX and SlSOS4 genes and the amplified fragments were cloned into the vector pYL156 (Liu et al. 2002). The recombinant vectors pTRVPDX1.2, pTRV-PDX1.3, pTRV-PDX2, and pTRV-SOS4 were confirmed by enzyme digestion and then introduced into Agrobacterium tumefaciens GV3101 by electroporation using the GENE PULSER II Electroporation System (Bio-Rad Laboratories). A negative silencing vector containing a $G U S$ gene fragment, pTRV-GUS, and a positive silencing vector containing a PDS gene fragment, pTRV-PDS, were also constructed and used in the VIGS experiments. A. tumefaciens carrying different recombinant vectors was grown with shaking at $200 \mathrm{rpm}$ in YEP (1\% yeast extract, $1 \%$ tryptone, and $0.5 \% \mathrm{NaCl}, \mathrm{pH} 7.2)$ medium with kanamycin at $50 \mu \mathrm{g} / \mathrm{ml}$, rifampicin at $50 \mu \mathrm{g} / \mathrm{ml}$, and gentamycin at $25 \mu \mathrm{g} / \mathrm{ml}$ in a $28^{\circ} \mathrm{C}$ shaker. Cells were harvested by centrifugation at $4,000 \times g$ and $4^{\circ} \mathrm{C}$ for $10 \mathrm{~min}$ and resuspended to a final concentration to an optical density at $600 \mathrm{~nm}\left(\mathrm{OD}_{600}\right)=1.5$ in a solution containing $10 \mathrm{mM} \mathrm{MgCl}$, $10 \mathrm{mM}$ morpholinoethanesulfonic acid ( $\mathrm{pH}$ 5.7), and $150 \mu \mathrm{M}$ acetosyringone and kept in the dark for at least $3 \mathrm{~h}$ at room temperature before infiltration (Hartl et al. 2008). Bacterial suspension containing a different pTRV2 construct was mixed with the same volume of agrobacteria containing pTRV1 (pYL196) (Liu et al. 2002). The mixed agrobacterial suspension was infiltrated into the abaxial surface of the 2-week-old seedlings using a 1-ml needleless syringe. Efficiency of the VIGS protocol was evaluated using a $P D S$ gene as a marker of silencing in tomato plants, according to Liu and associates (2002). Single VB6 biosynthetic gene-silenced plants in a set of experiments with $100 \%$ of pTRV-PDS-silenced plants showing bleaching phenotype were used for disease and biochemical assays. Leaf samples were collected at 20 days after VIGS infiltration and used for analysis of silencing efficiency by qRTPCR.

\section{Disease assays.}

B. cinerea was grown on V8 medium ( $36 \%$ V8 juice, $0.2 \%$ $\mathrm{CaCO}_{3}$, and $2 \%$ agar) at room temperature. Spores were collected by passing through two layers of cheesecloth in $4 \%$ maltose and $1 \%$ peptone buffer, and the spore density was adjusted to $1 \times 10^{5}$ spores $/ \mathrm{ml}$. Four-week-old plants were inoculated by foliar spraying with the spore suspension. Alternatively, fully expanded leaves detached from tomato plants were placed onto water-saturated filter paper and inoculated by a drop inoculation method according to a previously reported procedure (AbuQamar et al. 2008). The inoculated plants and detached leaves were covered with a transparent plastic film and kept in a growth chamber with similar conditions as for plant growth. Fungal growth in planta was determined by analyzing $B$. cinerea ActinA transcripts by qRT-PCR. Lesion size was measured 3 dpi. Leaves from at least 10 individual plants were used in each independent experiment.

\section{Measurement of VB6 content.}

A bioassay with a yeast strain (ATCC 9080) auxotrophic for VB6 was used to measure total VB6 content in the leaf tissues. Leaf extracts were prepared with a protocol, as described previously (Denslow et al. 2005). After extraction, $\mathrm{pH}$ values of the samples were adjusted to 4.8 and freshly prepared $\beta$-glycosidase $(5 \mathrm{mg} / \mathrm{ml})$ and acid phosphatase $(37.5 \mathrm{mg} / \mathrm{ml})$ was added to deglycosylate and dephosphorylate VB6 vitamers. These leaf extracts in 50-ml falcon tubes were incubated overnight at $37^{\circ} \mathrm{C}$ with shaking at $70 \mathrm{rpm}$ and then filter sterilized using a 50-ml Millipore's Steriflip Filter Unit $(0.22 \mu \mathrm{M})$. Yeast cells grown on YPD (1\% yeast extract, $2 \%$ peptone and $2 \%$ dextrose) overnight at $30^{\circ} \mathrm{C}$ were washed twice with sterile distilled water and pelleted by centrifuging at $4^{\circ} \mathrm{C}$ and $1,000 \times g$ for $10 \mathrm{~min}$. For bioassay of VB6 content, 20-ml falcon tubes containing $5 \mathrm{ml}$ of pyridoxol Y medium (HB8684; Hope BioTechnology Co., Qingdao, China) were mixed with $5 \times 10^{6}$ yeast cells and $1 \mathrm{ml}$ of treated leaf extract. A standard curve was made using the same protocol with $0,1,2,3,4,5,6,7,8$, and $9 \mathrm{ng}$ of total PN. All test and standard samples were run in triplicate and incubated at $30^{\circ} \mathrm{C}$ with shaking at $250 \mathrm{rpm}$. Yeast growth was measured at 17 and $24 \mathrm{~h}$ with a spectrophotometer at $\mathrm{OD}_{540}$. The total VB6 content in leaf extracts was calculated by comparison with the standard curve.

\section{Yeast complementation assays.}

Yeast (Saccharomyces cerevisiae) strains BQS1037 and MML21, which are defective in ScSNZ1 and ScSNO1, respectively, were used (Rodríguez-Navarro et al. 2002; TambascoStudart et al. 2005). The coding sequences of SIPDXI and SIPDX2 genes were amplified using gene-specific primers (Table 1) and then cloned into pYES2. The recombinant plasmids containing SIPDX1.2/1.3 or SIPDX2 gene were transformed into BQS1037 and MML21, respectively. Complementation was carried out on SC medium with or without VB6 supplement and with the appropriate selection markers (TambascoStudart et al. 2005).

\section{In situ detection and quantitative measurement of ROS.}

In situ detection of superoxide anion in leaf tissues was performed by nitroblue tetrazolium (NBT) staining (Doke et al. 1983). Leaf samples were collected at 0 (as mock-inoculation control) or 48 hpi with $B$. cinerea and were vacuum infiltrated in $10 \mathrm{mM}$ potassium phosphate buffer, $\mathrm{pH} 7.5$, containing 10 $\mathrm{mM} \mathrm{NaN}_{3}$ and $0.1 \%$ NBT (Sigma, St. Louis) for $30 \mathrm{~min}$ at room temperature. Leaves were boiled in $96 \%$ ethanol for 10 min before visualizing blue precipitates. Quantitative measurement of $\mathrm{H}_{2} \mathrm{O}_{2}$ was performed using hydrogen peroxide detection kits (number 064; Jiancheng Bioengineering Institute, Nanjing, China) according to the manufacturer's protocol. The content of $\mathrm{H}_{2} \mathrm{O}_{2}$ was determined spectrophotometrically by measuring $\mathrm{OD}_{405}$ based on the formation of complexes from reaction of molybdate with $\mathrm{H}_{2} \mathrm{O}_{2}$ in the samples and calculated according to a standard curve prepared with the same protocol.

\section{Measurements of enzyme activity and antioxidant compounds.}

Leaf samples were collected at 0 (as mock-inoculation controls), 24 , or 48 hpi with $B$. cinerea and then subjected to prepare leaf extracts for biochemical assays. Activities of total SOD and CAT, RSC, and contents of GSH and GSSG were measured by standard colorimetric assays using commercial kits (Jiancheng Bioengineering Institute), according to the manufacturer's protocols. Briefly, SOD activity was determined colorimetrically by measuring $\mathrm{OD}_{530}$ using the superoxide dismutase detection kit (number A001-1), which is based on the oxidation of hydroxylamine to form purple products by 
superoxide anions generated from the system of xanthine and xanthine oxidase. One unit of SOD was defined as the amount of SOD required to inhibit the oxidation of hydroxylamine by $50 \%$. Activity of CAT was determined using the catalase detection kit (number A007-2), which measured the consumption of $\mathrm{H}_{2} \mathrm{O}_{2}$ at $\mathrm{OD}_{240}$ for $1 \mathrm{~min}$. The contents of GSH and GSSG were determined using the oxidative glutathione detection kit (number A061-2). GSSG in the samples was first reduced to GSH by glutathione reductase and GSH was determined by measuring $\mathrm{OD}_{412}$ using 5,5-dithio-bis-2-nitrobenzoic acid for color development. RSC was assumed colorimetrically using the superoxide anion detection kit (number A052) by measuring $\mathrm{OD}_{550}$, representing the ability of the samples to inhibit the reduction of NBT by superoxide anion generated from reactions of xanthine and xanthine oxidase. Protein concentrations in all samples were determined by the Coomassie brilliant blue method (Jiancheng Bioengineering Institute).

\section{Statistical analysis.}

All experiments were performed in triplicate and all data obtained were subjected to statistical analysis according to the Student's $t$ test and the probability values of $P<0.05$ were considered to be significant.

\section{ACKNOWLEDGMENTS}

This work was supported by the National Basic Research Program of China (2009CB119005), the National Key Technology R \& D Program of China (2011BAD12B04), the Ph.D. Program Foundation for Young Teachers of Ministry of Education of China (number 20100101120079), and the Scientific Research Fund of Department of Education of Zhejiang Province (number Y200909712).

\section{LITERATURE CITED}

AbuQamar, S., Chai, M. F., Luo, H., Song, F., and Mengiste, T. 2008. Tomato protein kinase $1 \mathrm{~b}$ mediates signaling of plant responses to necrotrophic fungi and insect herbivory. Plant Cell 20:1964-1983.

Bilski, P., Li, M. Y., Ehrenshaft, M., Daub, M. E., and Chignell, C. F. 2000. Vitamin $B_{6}$ (pyridoxine) and its derivatives are efficient singlet oxygen quenchers and potential fungal antioxidants. Photochem. Photobiol. 71:129-134.

Chen, H., and Xiong, L. 2005. Pyridoxine is required for post-embryonic root development and tolerance to osmotic and oxidative stress. Plant J. 44:396-408.

Danon, A., Miersch, O., Felix, G., Camp, R. G. L., and Apel, K. 2005. Concurrent activation of cell death-regulating signaling pathways by singlet oxygen in Arabidopsis thaliana. Plant J. 41:68-80.

Denslow, S. A., Walls, A. A., and Daub, M. E. 2005. Regulation of biosynthetic genes and antioxidant properties of vitamin $\mathrm{B}_{6}$ vitamers during plant defense responses. Physiol. Mol. Plant Pathol. 66:244255.

Denslow, S. A., Rueschhoff, E. E., and Daub, M. E. 2007. Regulation of the Arabidopsis thaliana vitamin $\mathrm{B}_{6}$ biosynthesis genes by abiotic stress. Plant Physiol. 45:152-161.

Doke, N. 1983. Generation of superoxide anion by potato-tuber protoplasts during the hypersensitive response to hyphal wall components of Phytophthora infestans and specific inhibition of the reaction by suppressors of hypersensitivity. Physiol. Plant Pathol. 23:359-367.

Dong, Y. X., Sueda, S., Nikawa, J. I., and Kondo, H. 2004. Characterization of the products of the genes SNO1 and SNZ1 involved in pyridoxine synthesis in Saccharomyces cerevisiae. Eur. J. Biochem. 271:745752 .

Drewke, C., and Leistner, E. 2001. Biosynthesis of vitamin $\mathrm{B}_{6}$ and structurally related derivatives. Vitam. Horm. 61:121-155.

González, E., Danehower, D., and Daub, M. E. 2007. Vitamer levels, stress response, enzyme activity, and gene regulation of Arabidopsis lines mutant in the pyridoxine/pyridoxamine $5^{\prime}$-phosphate oxidase $(P D X 3)$ and the pyridoxal kinase(SOS4) genes involved in the vitamin $\mathrm{B}_{6}$ salvage pathway. Plant Physiol. 145:985-996.

Hartl, M., Merker, H., Schmidt, D. D., and Baldwin, I. T. 2008. Optimized virus-induced gene silencing in Solanum nigrum reveals the defensive function of leucine aminopeptidase against herbivores and the shortcomings of empty vector controls. New Phytol. 179:356-365.
Havaux, M., Ksas, B., Szewczyk, A., Rumeau, D., Franck, F., Caffarri, S., and Triantaphylidès, C. 2009. Vitamin B6 deficient plants display increased sensitivity to high light and photo-oxidative stress. BMC Plant Biol. 9:130.

Herrero, S., González, E., Gillikin, J. W., Vélëz, H., and Daub, M. E. 2011 Identification and characterization of a pyridoxal reductase involved in the vitamin B6 salvage pathway in Arabidopsis. Plant Mol. Biol. 76:157-169.

Hill, R. E., and Spenser, I. D. 1996. Biosynthesis of vitamin B6. Cell. Mol. Biol. 2:695-703.

Kuźniak, E., and Skłodowska, M. 2004. The effect of Botrytis cinerea infection on the antioxidant profile of mitochondria from tomato leaves. J. Exp. Bot. 55:605-612.

Kuźniak, E., and Skłodowska, M. 2005a. Compartment-specific role of the ascorbate-glutathione cycle in the response of tomato leaf cells to $\mathrm{Bo}$ trytis cinerea infection. J. Exp. Bot. 56:921-933.

Kuźniak, E., and Skłodowska, M. 2005b. Fungal pathogen-induced changes in the antioxidant systems of leaf peroxisomes from infected tomato plants. Planta 222:192-200.

Leuendorf, J. E., Osorio, S., Szewczyk, A., Fernie, A. R., and Hellmann, H. 2010. Complex assembly and metabolic profiling of Arabidopsis thaliana plants overexpressing vitamin $\mathrm{B}_{6}$ biosynthesis proteins. Mol. Plant 3:890-903.

Liu, Y., Schiff, M., and Dinesh-Kumar, S. P. 2002. Virus-induced gene silencing in tomato. Plant J. 31:777-786.

Livak, K. J., and Schmittgen, T. D. 2001. Analysis of relative gene expression data using real-time quantitative PCR and the $2^{-\Delta \Delta} \mathrm{CT}$ method. Methods 25:402-408.

Lum, H. K., Kwok, F., and Lo, S. C. L. 2002. Cloning and characterization of Arabidopsis thaliana pyridoxal kinase. Planta 215:870-879.

McCormick, D. B., and Chen, H. 1999. Update on interconversions of vitamin B-6 with its coenzyme. J. Nutr. 129:325-327.

Mengiste, T. 2012. Plant immunity to necrotrophs. Annu. Rev. Phytopathol. 50:267-294.

Mengiste, T., Chen, X., Salmeron, J., and Dietrich, R. 2003. The BOTRYTIS SUSCEPTIBLE1 gene encodes an R2R3MYB transcription factor protein that is required for biotic and abiotic stress responses in Arabidopsis. Plant Cell 15:2551-2565.

Mittenhuber, G. 2001. Phylogenetic analyses and comparative genomics of vitamin B6 (pyridoxine) and pyridoxal phosphate biosynthesis pathways. J. Mol. Microbiol. Biotechnol. 3:1-20.

Mooney, S., Leuendorf, J. E., Hendrickson, C., and Hellmann, H. 2009 Vitamin B6: A long known compound of surprising complexity. Molecules 14:329-351.

Mooney, S., Chen, L., Kühn, C., Navarre, R., Knowles, N. R., and Hellmann, H. 2013. Genotype-specific changes in vitamin B6 content and the PDX family in potato. Biomed. Res. Int. 2013:389723.

Raschke, M., Boycheva, S., Crèvecoeur, M., Nunes-Nesi, A., Witt, S., Fernie, A. R., Amrhein, N., and Fitzpatrick, T. B. 2011. Enhanced levels of vitamin $\mathrm{B}_{6}$ increase aerial organ size and positively affect stress tolerance in Arabidopsis. Plant J. 66:414-432.

Ristilä, M., Strid, H., Eriksson, L. A., Strid, A., and Sävenstrand, H. 2011. The role of the pyridoxine (vitamin $\mathrm{B}_{6}$ ) biosynthesis enzyme PDX1 in ultraviolet-B radiation responses in plants. Plant Physiol. Biochem. 49:284-292.

Rodríguez-Navarro, S., Llorente, B., Rodríguez-Manzaneque, M. T., Ramne, A., Uber, G., Marchesan, D., Dujon, B., Herrero, E., Sunnerhagen, P., and Pérez-Ortín, J. E. 2002. Functional analysis of yeast gene families involved in metabolism of vitamins $B_{1}$ and $B_{6}$. Yeast 19:1261-1276.

Rueschhoff, E. E., Gillikin, J. W., Sederoff, H. W., and Daub, M. E. 2013. The SOS4 pyridoxal kinase is required for maintenance of vitamin $\mathrm{B}_{6}$ mediated processes in chloroplasts. Plant Physiol. Biochem. 63:281291.

Sang, Y., Barbosa, J. M., Wu, H., Locy, R. D., and Singh, N. K. 2007. Identification of a pyridoxine (pyridoxamine) 5'-phosphate oxidase from Arabidopsis thaliana. FEBS (Fed. Eur. Biochem. Soc.) Lett. 581:344-348.

Sang, Y., Locy, R. D., Goertzen, L. R., Rashotte, A. M., Si, Y., Kang, K., and Singh, N. K. 2011. Expression, in vivo localization and phylogenetic analysis of a pyridoxine 5'-phosphate oxidase in Arabidopsis thaliana. Plant Physiol. Biochem. 49:88-95.

Shi, H., and Zhu, J. K. 2002. SOS4, a pyridoxal kinase gene, is required for root hair development in Arabidopsis. Plant Physiol. 129:585-593.

Shi, H., Xiong, L., Stevenson, B., Lu, T., and Zhu, J. K. 2002. The Arabidopsis salt overly sensitive 4 mutants uncover a critical role for vitamin B6 in plant salt tolerance. Plant Cell 14:575-588.

Stocker, P., Lesgards, J. F., Vidal, N., Chalier, F., and Prost, M. 2003. ESR study of a biological assay on whole blood: Antioxidant efficiency of various vitamins. Biochim. Biophys. Acta 1621:1-8

Tambasco-Studart, M., Titiz, O., Raschle, T., Forster, G., Amrhein, N., and 
Fitzpatrick, T. B. 2005. Vitamin B6 biosynthesis in higher plants. Proc. Natl. Acad. Sci. U.S.A. 102:13687-13692.

Tambasco-Studart, M., Tews, I., Amrhein, N., and Fitzpatrick, T. B. 2007. Functional analysis of PDX2 from Arabidopsis, a glutaminase involved in vitamin B6 biosynthesis. Plant Physiol. 144:915-925.

Titiz, O., Tambasco-Studart, M., Warzych, E., Apel, K., Amrhein, N., Laloi, C., and Fitzpatrick, T. B. 2006. PDX1 is essential for vitamin B6 biosynthesis, development and stress tolerance in Arabidopsis. Plant J. 48:933-946

Vanderschuren, H., Boycheva, S., Li, K. T., Szydlowski, N., Gruissem, W., and Fitzpatrick, T. B. 2013. Strategies for vitamin B6 biofortification of plants: A dual role as a micronutrient and a stress protectant. Front. Plant Sci. 4:143.

Veronese, P., Nakagami, H., Bluhm, B., Abuqamar, S., Chen, X., Salmeron, J., Dietrich, R. A., Hirt, H., and Mengiste, T. 2006. The membraneanchored BOTRYTIS-INDUCED KINASE1 plays distinct roles in Arabidopsis resistance to necrotrophic and biotrophic pathogens. Plant Cell $18: 257-273$

Wagner, S., Bernhardt, A., Leuendorf, J. E., Drewke, C., Lytovko, A.,
Mujahed, N., Gurgui, C., Frommer, W. B., Leistner, E., Fernie, A. R and Hellmann, H. 2006. Analysis of the Arabidopsis rsr4-1/pdx1-3 mutant reveals the critical function of the PDX1 protein family in metabolism, development, and vitamin B6 biosynthesis. Plant Cell 18:1722-1735.

Wetzel, D. K., Ehrenshaft, M., Denslow, S. A., and Daub, M. E. 2004. Functional complementation between the PDX1 vitamin $\mathrm{B}_{6}$ biosynthetic gene of Cercospora nicotianae and pdxJ of Escherichia coli. FEBS (Fed. Eur. Biochem. Soc.) Lett. 564:143-146.

Zhang, Y., Butelli, E., De Stefano, R., Schoonbeek, H. J., Magusin, A., Pagliarani, C., Wellner, N., Hill, L., Orzaez, D., Granell, A., Jones, J. D., and Martin, C. 2013. Anthocyanins double the shelf life of tomatoes by delaying overripening and reducing susceptibility to gray mold. Curr. Biol. 23:1094-100.

\section{AUTHOR-RECOMMENDED INTERNET RESOURCE}

Sol Genomics database: solgenomics.net 